\title{
Exergames for women with fibromyalgia. A randomised controlled trial to evaluate the effects on mobility skills, balance and fear of falling.
}

\author{
Daniel Collado-Mateo ${ }^{\text {Corresp., }}{ }^{1}$, Francisco J Dominguez-Muñoz ${ }^{1}{ }^{\text {, }}$ Jose C Adsuar ${ }^{1}$, Eugenio Merellano-Navarro ${ }^{2}$, \\ Narcis Gusi ${ }^{1}$ \\ ${ }^{1}$ Faculty of Sport Science. Departamento de Didáctica de la Expresión Musical, Plástica y Corporal., University of Extremadura, Cáceres, Spain \\ 2 Facultad de Educación., Universidad Autónoma de Chile, Talca, Chile \\ Corresponding Author: Daniel Collado-Mateo \\ Email address: dcolladom@unex.es
}

Background: Exergames are a new form of rehabilitation that combine the characteristics of physical exercise and the benefits of non-immersive virtual reality (VR). Effects of this novel therapy in women fibromyalgia are still unknown. The objective was to evaluate the effects of exergame-based intervention on mobility skills, balance and fear of falling in women with fibromyalgia. Methods: This study was a randomized controlled trial with concealed allocation. Seventy-six women with fibromyalgia were divided into two groups: the exercise group received an 8 week intervention based on exergames, while the control group continued their usual activities. Mobility skills were evaluated using the timed up and go test, while balance was assessed using the functional reach test, and the CTSIB protocol. Fear of falling was evaluated on a scale of 0-100 (0, no fear; 100, extreme fear). Measurements were performed before and after the intervention. A repeated-measures linear mixed model was used to compare the effects of the intervention between the two groups. Results: The exercise group was significantly quicker than the control group in the timed up and go test (MD, $-0.71 ; 95 \% \mathrm{Cl},-1.09-0.32 ; \mathrm{p}<0.001$ ). There were also significant improvements in functional reach and a reduced fear of falling (MD, 4.34; 95\% $\mathrm{Cl}, 1.39-7.30 ; p=0.005$ and $\mathrm{MD},-9.85 ; 95 \% \mathrm{Cl},-0.19--0.08 ; p=0.048$, respectively). Discussion: The improved TUG observed herein was better than the smallest real difference. Based on the results on mobility skills, balance and fear of falling, exergames may be an effective tool as a therapy for women with fibromyalgia. 


\section{Exergames for women with fibromyalgia. A randomised controlled trial to}

2 evaluate the effects on mobility skills, balance and fear of falling.

3 Daniel Collado-Mateo ${ }^{1}$, Francisco J. Dominguez-Muñoz ${ }^{2}$, José C. Adsuar ${ }^{3}$, Eugenio Merellano-Navarro ${ }^{4}$,

4 Narcis Gusi ${ }^{5}$.

5 1. Faculty of Sport Science, University of Extremadura, Cáceres, Spain, dcolladom@unex.es

6 2. Faculty of Sport Science, University of Extremadura, Cáceres, Spain, fjavierdominguezm@gmail.com

7 3. Faculty of Sport Science, University of Extremadura, Cáceres, Spain, jadssal@unex.es

8 4. Facultad de Educación, Universidad Autónoma de Chile, Talca, Chile. emerellanon@uautonoma.cl

9 5. Faculty of Sport Science, University of Extremadura, Cáceres, Spain, ngusi@unex.es

Corresponding author: Daniel Collado-Mateo, Faculty of Sport Science, University of 


\section{ABSTRACT}

Background: Exergames are a new form of rehabilitation that combine the characteristics of physical exercise and the benefits of non-immersive virtual reality (VR). Effects of this novel therapy in women fibromyalgia are still unknown. The objective was to evaluate the effects of exergame-based intervention on mobility skills, balance and fear of falling in women with fibromyalgia.

Methods: This study was a randomized controlled trial with concealed allocation. Seventy-six women with fibromyalgia were divided into two groups: the exercise group received an 8 week intervention based on exergames, while the control group continued their usual activities. Mobility skills were evaluated using the timed up and go test, while balance was assessed using the functional reach test, and the CTSIB protocol. Fear of falling was evaluated on a scale of 0-100 $(0$, no fear; 100 , extreme fear). Measurements were performed before and after the intervention. A repeated-measures linear mixed model was used to compare the effects of the intervention between the two groups.

Results: The exercise group was significantly quicker than the control group in the timed up and go test (MD, $-0.71 ; 95 \% \mathrm{CI},-1.09-0.32 ; \mathrm{p}<0.001)$. There were also significant improvements in functional reach and a reduced fear of falling $(\mathrm{MD}, 4.34 ; 95 \% \mathrm{CI}, 1.39-7.30 ; \mathrm{p}=0.005$ and $\mathrm{MD}$, 9.85; 95\% CI, -0.19--0.08; $\mathrm{p}=0.048$, respectively).

Discussion: The improved TUG observed herein was better than the smallest real difference. Based on the results on mobility skills, balance and fear of falling, exergames may be an effective tool as a therapy for women with fibromyalgia. 
42

43

44

\section{INTRODUCTION}

Fibromyalgia (FM) is a chronic syndrome characterized by widespread pain and other symptoms such as fatigue, trouble sleeping, anxiety, depression, impaired balance, stiffness, a high risk of falling, and poor physical fitness (Walker, 2016; Wolfe et al., 2010), all of which have a significant impact on quality of life (Huijnen, Verbunt, Meeus, \& Smeets, 2014). The estimated overall prevalence of FM in the general European population ranges from 2.9\% to 4.7\% (Branco et al., 2010), while it is lower in the United States (about 1.75\%; 95\% CI, 1.42-2.07) (Walitt, Nahin, Katz, Bergman, \& Wolfe, 2015). FM mostly affects women, who often experience higher levels of pain. On the other hand, men show higher disability and reduced physical function (CastroSanchez et al., 2012). Furthermore, men and women show different responses to therapy (Lami et al., 2016).

Treatment of fibromyalgia typically involves a combination of pharmacological and nonpharmacological approaches. According to (Walker, 2016) the main objectives are to improve the ability to perform activities of daily living by increasing physical function and reducing pain levels, which often lead to an improvement in quality of life. Exercise is usually the first step of a multidisciplinary approach (Walker, 2016). The effects of exercise on physical function (including balance and mobility skills) of women with FM are well documented. However, the effect size ranges from small to large depending on the type of exercise and the outcome measure used (Bidonde, Busch, Webber, et al., 2014). Although therapies based on whole body vibration (Adsuar, Del Pozo-Cruz, Parraca, Olivares, \& Gusi, 2012), aquatic training (Tomas-Carus et al., 
63 2009), or balance exercise training (Duruturk, Tuzun, \& Culhaoglu, 2015) effectively improve

64 static and dynamic balance in women with FM, adherence to these exercise-based interventions is

65 frequently poor (Oliver \& Cronan, 2002); therefore, more motivating and enjoyable alternatives

66 are required (Mortensen, Kristensen, Brooks, \& Brooks, 2015).

67

68

Exergames are a form of rehabilitation that combine the characteristics of physical exercise and the benefits of non-immersive virtual reality (VR) (Skip Rizzo, Lange, Suma, \& Bolas, 2011), such as distraction from pain or enjoyment (Mortensen et al., 2015). This therapeutic modality has been used in older adults to improve fear of falling and balance during quiet standing under eyes open and eyes closed conditions (Gschwind et al., 2015; van Diest et al., 2016). Exergames may be a motivating and enjoyable alternative form of rehabilitation (Burke et al., 2009; Herrero, Garcia-Palacios, Castilla, Molinari, \& Botella, 2014; Mortensen et al., 2015). However, to the best of our knowledge, no randomized controlled trial has examined the effects of exergames on patients with FM.

Therefore, the objective of this randomized controlled trial was to evaluate the effects of exergamebased intervention on mobility skills, balance and fear of falling in women with fibromyalgia. 
84 This randomized controlled trial was registered in the Australian New Zealand Clinical Trials

85 Registry, ACTRN12615000836538. Two groups were included: an exercise group (EG) and a

86

87

88

89

90

91

92

93

94

95

96

97

98

control group (CG). All patients were provided with written informed consent to participate and the study protocol was approved by the Committee of Bioethics of the University of Extremadura in May 2015 (reference number 61/2015). Randomisation and group allocation were performed by a single researcher. Allocation was concealed. Randomisation was based on computergenerated random code numbers. Half of the numbers were randomly assigned to the $\mathrm{CG}$ and the rest were assigned to the EG. The researchers in charge of evaluation were blind to this process. Participants were not blinded as they had been informed about the protocol and knew whether or not they were performing physical exercise.

\section{Participants}

The sample size was calculated to assess the effects of the intervention on impact of FM. This variable is often assessed using the FM impact questionnaire (FIQ) (R. Bennett, 2005). The minimum clinically important reduction in the FIQ was set at $14 \%$ of the total FIQ score, as described previously (R. M. Bennett, Bushmakin, Cappelleri, Zlateva, \& Sadosky, 2009). For calculation purposes, the estimated mean FIQ was $70.5 \pm 11.8$, which was based on data from a previous study of a Spanish cohort (Esteve-Vives, Rivera Redondo, Isabel Salvat Salvat, de Gracia Blanco, \& de Miquel, 2007). Initially, the minimum power for detecting between-group differences of at least $14 \%$ was set at $85 \%$, with an $\alpha$ value of 0.05 . Based on these parameters, a minimum of 26 participants per group was necessary. No maximum number was set. A total of 83 women with FM fulfilled the inclusion criteria and were randomly assigned to the two groups. A maximum loss to follow-up of $15 \%$ was assumed. With respect to the main outcome measure (i.e., 
107 the TUG), the mean, standard deviation (SD), and smallest real difference were extracted from a 108 previous study based on Spanish women with FM (Collado-Mateo et al., 2016). Assuming a mean 109 (SD) of $6.791(0.830)$, a difference of at least 9.33\% (smallest real difference), with a power of $11088 \%$ and an $\alpha$ value of 0.05 based on two groups (EG and CG), could be detected using a sample 111 size of 35 participants per group.

112 Recruitment started in November 2015 and final measurements were carried out in May 2016.

113 Recruitment was performed at two local FM associations. The inclusion criteria were as follows:

114 a) female, b) aged 30-75 years, c) diagnosed with FM by a rheumatologist according to the 115 criteria of the American College of Rheumatology (Wolfe et al., 2010; Wolfe et al., 1990), d)

116 able to communicate effectively with the study staff, and e) had read, understood, and signed the 117 written informed consent form. Participants were excluded if they: a) were pregnant, b) changed 118 their usual care therapies during the 8 weeks of treatment, and, c) had contraindications for 119 physical exercise. Participants could abandon the study if a) they withdrew informed consent, b) the researcher or their general practitioner felt that they should withdraw from the study for

121 reasons of safety, or c) the participant did not attend at least $75 \%$ of the sessions.

\section{Intervention}

The intervention comprised 8 weeks of VR-based physical exercise. The EG performed this activity twice a week for 1 hour per session, whereas the CG received no treatment and continued their usual activities. All sessions were performed at the local association's facilities. To increase the motivational and social component, exercises were performed in groups of three, with each person interacting with their own device. Intervention was based on an exergame called VirtualExFM, which was designed by the research group to improve physical conditioning of women with 
130 FM and increase their ability to perform activities of daily living. This exergame is based on

131 Microsoft Kinect ${ }^{\circledR}$, which connects to a computer and serves as the input sensor for tracking the

132 movements of the participant. The software is run in the computer and contains a control panel

133 through which the targets and difficulty can be modified. The system provides immediate visual

134 feedback about the extent their movements fit the required patterns.

135 VirtualEx-FM provides three different virtual environments:

a) The first comprises a warm-up followed by the aerobic part of the session. The participant has to imitate the movements that appear on the screen, which are performed by a professional kinesiologist and dance teacher, recorded, and stored in a software directory. Files can be removed, added, and modified as needed. Additionally, the playback speed can be manually controlled $(0.5 \times, 1 \times, 1.5 \times$, and $2 \times)$. In general, there were two types of video: warm-up videos based on full body joint movements, and dancing videos. The latter were based on Zumba, which is a fusion of aerobics and salsa.

b) The second environment focuses on postural control and coordination. Participants interact with an apple that appears and disappears around them. The position at which the apple appears can be manually programmed by the technician. The sequence of which parts of the body interact with the apple can also be controlled and modified.

c) The aim of the third environment is to improve mobility skills, balance and coordination. Participants are asked to step on virtual footprints. The distance between the footprints is controlled by the technician. The interface allows the technician to choose different types of steps: normal, tiptoe, heel-walking, raising the knees, and raising the heels. 
152 The preferences (Lewis \& Rosie, 2012) and needs (Bidonde, Busch, Bath, \& Milosavljevic, 2014)

153 of women with FM were considered when training in the three environments. Furthermore,

154 VirtualEx-FM fulfilled the eight key points proposed by Lewis and Rosie (2012) for enhancing

155 the efficacy of VR-based systems to optimize the rehabilitation benefits. These key points include

156 all aspects related to the interface, social interaction, therapeutically principled movement patterns,

157 progressively challenging games, and immediate feedback.

158

159 Outcome variables

160 All evaluations were performed in a laboratory at the university. Baseline data included age, years

161 since FM diagnosis, BMI, and impact of FM as assessed by the revised version of the FM impact

162 questionnaire (FIQ-R) (R. M. Bennett, Friend, et al., 2009).

163

164

Primary outcome

165 The timed up and go (TUG) (Podsiadlo \& Richardson, 1991) test was used to assess mobility 166 skills. The time taken by participants to rise from a chair, walk $3 \mathrm{~m}$, turn around, walk back to the 167 chair, and sit down again was measured. The best of three repetitions was recorded.

168

169 Secondary outcomes

170 Other variables were balance and fear of falling. Balance was evaluated using the functional reach

171 (FR) and the Clinical Test of Sensory Integration of Balance (CTSIB) tests (Di Fabio \& Seay, 172 1997). Fear of falling was assessed on a visual analogue scale, ranging from 0 to 100 , where 0 was 
173 "no fear" and 100 was “extreme fear" (Scheffer, Schuurmans, vanDijk, van der Hooft, \& de Rooij, 174 2010).

175

176 The CTSIB is a widely used balance protocol and was conducted using the Biodex Balance System

177 (Shirley, NY, USA). The test comprises four conditions: eyes open on a firm surface, eyes closed 178 on a firm surface, eyes open on an unstable surface, and eyes closed on an unstable surface.

179 Participants have to maintain their feet on the platform during the $30 \mathrm{~s}$ test, with a rest of 10 seconds 180 between the different conditions. Higher sway indices would mean poorer balance. The FR 181 (Duncan, Weiner, Chandler, \& Studenski, 1990) measures the maximum distance participants can 182 reach forward beyond arms' length while keeping their feet on the floor, hip width apart. Two 183 repetitions were performed and the best of them was analysed.

\section{Data analysis}

185 The mean effect (SD) for each group was calculated by subtracting the pre-intervention scores

186 from the post-intervention scores. Between-group changes were calculated by subtracting the 187 effects reported for the CG from the effects observed for the EG. A repeated-measures linear mixed 188 model was used to compare the effects of the intervention between groups. Effect size was 189 considered as low $(<0.2)$, medium $(>0.2$ and $<0.8)$ or large $(>0.8)$ (Cohen, 1988).

191 Each group was divided into two subgroups to identify differences between women with and without fear of falling. For that purpose, the cut-off point was set at 50 because a previous study reported a mean of 48.88 in the VAS for women with fibromyalgia (Collado-Mateo et al., 2015). 


\section{RESULTS}

196 The flow of participants is shown in Figure 1. In total, 86 women were screened and three were 197 excluded for not meeting the inclusion criteria. These three women belonged to the association 198 and suffered from fatigue and pain, but they were not diagnosed with FM by a rheumatologist. 199 Therefore, 83 women were randomized into the EG $(n=42)$ and CG $(n=41)$. Table 1 summarizes 200 the main baseline characteristics of both groups.

201 Regarding the compliance with the treatment, forty-one of the forty-two women allocated to EG 202 completed the intervention, which means that $2.38 \%$ of women from this group were lost to follow 203 up. The cause of this loss was a change of residence. In the CG, 35/41 women attended the post204 intervention session, meaning a loss of $14.63 \%$. Intervention was considered complete when 205 participants attended a minimum of $75 \%$ of sessions. Only one of the researchers was aware of the 206 group to which each participant belonged. This researcher did not conduct the final measurements. 207 No adverse effects were noted.

Table 2 compares the groups in terms of primary and secondary outcomes. For the TUG, the time taken for the EG to complete the test at the end of the study was $0.49 \mathrm{~s}(\mathrm{SD}, 0.63)$ quicker than that at baseline. The time for the CG actually increased slightly (from $6.71 \mathrm{~s}$ at baseline to $6.93 \mathrm{~s}$ after the intervention). Therefore, the effect of exergames on the primary outcome was estimated as $-0.71(95 \% \mathrm{CI},-1.09-0.32)$, meaning a significant improvement of $10.61 \%(\mathrm{p}<0.001)$.

In the CTSIB protocol, the improvement was significantly higher for the EG only under the 215 condition of eyes closed on an unstable surface $(p=0.036)$. Results from the ANOVA almost 
216 reached significance for the condition of eyes closed on a stable surface condition and of eyes open

217 on an unstable surface ( $p=0.066$ and 0.055 , respectively). Significant improvement was observed

218 in the FR test $(p=0.005)$, the EG improved by $3.53 \mathrm{~cm}$ (an improvement of $23.58 \%)$, whereas the

219 CG deteriorated by $1.06 \mathrm{~cm}$.

221 Finally, the score for fear of falling in the EG fell from 26.10 to 21.09, while that in the CG 222 increased from 28.57 to 33.42 . Results from the ANOVA reached significance $(\mathrm{p}=0.048)$. As 223 shown in Table 3, the reduction was highest in participants with the greatest fear of falling. The 224 most fearful in the EG reduced their score from 63.46 to 43.84 , a reduction of $30 \%$.

\section{DISCUSSION}

227

228

229

230

231

232

233

234

235

236

237

This is the first randomized controlled trial to examine the utility of exergames as a therapy for improving mobility skills and balance in people with FM. These variables were improved through challenging exercises performed using a VR system. The improved TUG observed herein was slightly better than the smallest real difference reported in a previous study of women with FM (Collado-Mateo et al., 2016), which was reported as 9.33\%. Therefore, the improvement in mobility skills can be considered relevant. The rest of the measures assessed in the present study focused on static balance, since both the CTSIB protocol and the FR test are performed without displacement of the feet. The baseline sway indices for each condition in the CTSIB protocol were better than those reported in a previous study of women with FM (Collado-Mateo et al., 2015); the exception was the condition "eyes closed on an unstable surface". In the present study, this condition was significantly improved from $3.25^{\circ}$ at baseline (note that in the previous study, the 
238 mean sway index for women aged $>60$ was $3.29^{\circ}$ ) to $2.76^{\circ}$ (in the previous study, the mean value

239 for women with FM aged $<50$ was $2.77^{\circ}$ ). Therefore, balance performance under the condition of

240 standing with eyes closed on an unstable surface changed from $3.25^{\circ}$ (close to the mean for patients

241 aged $>60$ ) to $2.76^{\circ}$ (close to the mean for patients aged $<50$ ). The improvement in the FR test was

242 also significant, with a between-group change of more than $23 \%$.

244 Physical inactivity and a sedentary lifestyle are common in FM patients, which may be aggravated

245 by a greater fear of falling. This is especially relevant in this population, where around $72 \%$ are

246 overweight (Segura-Jimenez et al., 2015). By reducing the fear of falling, women with FM may

247 be able to increase their participation in physical, social, and other types of activity, thereby

248 improving their quality of life.

250 The impact of fear of falling on the activities of daily living of women with FM was examined by

251 Rutledge, Martinez, Traska, and Rose (2013): persons with FM often reported frustration because

252 they want to continue normal activities but are not able due to fear of falling. The reduction of fear

253 of falling in the EG was almost $20 \%$, which may be interpreted as a great improvement. In the

254 present study, fear of falling at baseline was lower than that reported in a previous study (Collado-

255 Mateo et al., 2015) in which the score ranged from 37 to 55. To explore this improvement more

256 deeply, the cohort was divided into two groups: women that scored $\geq 50$ on the fear of falling scale

257 and those that scored $<50$. Those in the EG with the greatest fear reduced their score from 63.46

258 to 43.84 , an improvement of almost 20 points. On the other hand, the score for the group with the

259 least fear increased from 8.75 to 10.53 . This was not observed for the CG, in which the 
260 improvement in the higher fear group was less than 5\%, while the score for the low fear group

261 increased by 7.4 points. Therefore, exergames have a large effect on fear of falling in women that

262 already experience higher levels of fear. By contrast, they have little effect on those with low levels

263 of fear.

264 The effect of age on balance and mobility skills is well documented. The present study included 265 women aged between 30 and 71, which is a wide range. Although the mean (SD) and distribution 266 of age was extremely similar in EG and CG, further attempts to evaluate the role of age on the 267 treatment effects were performed. First, "age" was included as a covariate in the model, which led 268 to only minimal changes in the p-values (see Table 2). Second, a correlation analysis of the 269 relationship between the treatment effects and age was performed. No statistically significant 270 correlation was observed. Therefore, the effects of exergame on mobility skills and balance seem 271 not to have been influenced by age in the current study. However, future studies should include 272 different age groups or restrict the age range.

273 Two main limitations should be mentioned. First, only women were included in the study due to 274 potential gender differences (Castro-Sanchez et al., 2012; Lami et al., 2016). Therefore, the effects

275 and applicability of exergame intervention on men with FM remain unknown. Second, the study 276 lacked an active CG. However, given the well-known effects of different types of exercise on FM, 277 effect sizes can be compared with those reported in the scientific literature.

279 CONCLUSIONS

280 Exergames are an effective tool for improving mobility skills, balance and fear of falling in women 281 with FM. The improvement in the TUG was better than the smallest real difference. Regarding 
282 balance, significant improvements were observed in FR and in the CTSIB with eyes closed on an

283 unstable surface. These results, along with high adherence (only one participant abandoned the

284 intervention), indicate that exergames may be a feasible alternative form of rehabilitation therapy

285 for improving balance and mobility problems in this population.

\section{REFERENCES}

288

289

290

291

292

293

294

295

296

297

298

299

300

301

302

303

Adsuar, J. C., Del Pozo-Cruz, B., Parraca, J. A., Olivares, P. R., \& Gusi, N. (2012). Whole body vibration improves the single-leg stance static balance in women with fibromyalgia: a randomized controlled trial. The Journal of sports medicine and physical fitness, 52(1), $85-91$

Bennett, R. (2005). The Fibromyalgia Impact Questionnaire (FIQ): a review of its development, current version, operating characteristics and uses. Clinical and experimental rheumatology, 23(5 Suppl 39), S154-162.

Bennett, R. M., Bushmakin, A. G., Cappelleri, J. C., Zlateva, G., \& Sadosky, A. B. (2009). Minimal clinically important difference in the fibromyalgia impact questionnaire. The Journal of rheumatology, 36(6), 1304-1311. doi: 10.3899/jrheum.081090

Bennett, R. M., Friend, R., Jones, K. D., Ward, R., Han, B. K., \& Ross, R. L. (2009). The Revised Fibromyalgia Impact Questionnaire (FIQR): validation and psychometric properties. Arthritis research \& therapy, 11(4), R120. doi: 10.1186/ar2783

Bidonde, J., Busch, A. J., Bath, B., \& Milosavljevic, S. (2014). Exercise for adults with fibromyalgia: an umbrella systematic review with synthesis of best evidence. Current rheumatology reviews, 10(1), 45-79. 
304 Bidonde, J., Busch, A. J., Webber, S. C., Schachter, C. L., Danyliw, A., Overend, T. J., . . .

305 Rader, T. (2014). Aquatic exercise training for fibromyalgia. The Cochrane database of systematic reviews(10), CD011336. doi: 10.1002/14651858.CD011336

307

308

309

310

311

312

313

314

315

316

317

318

319

320

321

322

323

324

325

326

Branco, J. C., Bannwarth, B., Failde, I., Abello Carbonell, J., Blotman, F., Spaeth, M., . . . Matucci-Cerinic, M. (2010). Prevalence of fibromyalgia: a survey in five European countries. Seminars in arthritis and rheumatism, 39(6), 448-453. doi:

10.1016/j.semarthrit.2008.12.003

Burke, J. W., McNeill, M., Charles, D. K., Morrow, P. J., Crosbie, J. H., \& McDonough, S. M. (2009). Optimising engagement for stroke rehabilitation using serious games. The Visual Computer, 25(12), 1085-1099.

Castro-Sanchez, A. M., Mataran-Penarrocha, G. A., Lopez-Rodriguez, M. M., Lara-Palomo, I. C., Arendt-Nielsen, L., \& Fernandez-de-las-Penas, C. (2012). Gender differences in pain severity, disability, depression, and widespread pressure pain sensitivity in patients with fibromyalgia syndrome without comorbid conditions. Pain medicine, 13(12), 1639-1647. doi: $10.1111 / j .1526-4637.2012 .01523 . x$

Cohen, J. (1988). Statistical power analysis for the behavioral sciences. 2nd edn. Mahwah, NJ: Academic press.

Collado-Mateo, D., Dominguez-Munoz, F. J., Adsuar, J. C., Merellano-Navarro, E., Olivares, P. R., \& Gusi, N. (2016). Reliability of the Timed Up and Go Test in Fibromyalgia. Rehabilitation nursing : the official journal of the Association of Rehabilitation Nurses. doi: $10.1002 /$ rnj.307

Collado-Mateo, D., Gallego-Diaz, J. M., Adsuar, J. C., Dominguez-Munoz, F. J., Olivares, P. R., \& Gusi, N. (2015). Fear of Falling in Women with Fibromyalgia and Its Relation with 
327

328

329 330

331

333

Number of Falls and Balance Performance. BioMed research international, 2015, 589014. doi: $10.1155 / 2015 / 589014$

Di Fabio, R. P., \& Seay, R. (1997). Use of the "fast evaluation of mobility, balance, and fear" in elderly community dwellers: validity and reliability. Physical therapy, 77(9), 904-917.

Duncan, P. W., Weiner, D. K., Chandler, J., \& Studenski, S. (1990). Functional reach: a new clinical measure of balance. J Gerontol, 45(6), M192-197.

Duruturk, N., Tuzun, E. H., \& Culhaoglu, B. (2015). Is balance exercise training as effective as aerobic exercise training in fibromyalgia syndrome? Rheumatology international, 35(5), 845-854. doi: 10.1007/s00296-014-3159-z

Esteve-Vives, J., Rivera Redondo, J., Isabel Salvat Salvat, M., de Gracia Blanco, M., \& de Miquel, C. A. (2007). [Proposal for a consensus version of the Fibromyalgia Impact Questionnaire (FIQ) for the Spanish population]. Reumatologia clinica, 3(1), 21-24. doi: $10.1016 / \mathrm{S} 1699-258 \mathrm{X}(07) 73594-5$

Gschwind, Y. J., Schoene, D., Lord, S. R., Ejupi, A., Valenzuela, T., Aal, K., . . Delbaere, K. (2015). The effect of sensor-based exercise at home on functional performance associated with fall risk in older people - a comparison of two exergame interventions. European review of aging and physical activity : official journal of the European Group for Research into Elderly and Physical Activity, 12, 11. doi: 10.1186/s11556-015-0156-5

Herrero, R., Garcia-Palacios, A., Castilla, D., Molinari, G., \& Botella, C. (2014). Virtual reality for the induction of positive emotions in the treatment of fibromyalgia: a pilot study over acceptability, satisfaction, and the effect of virtual reality on mood. Cyberpsychology, behavior and social networking, 17(6), 379-384. doi: 10.1089/cyber.2014.0052 
349 Huijnen, I. P., Verbunt, J. A., Meeus, M., \& Smeets, R. J. (2014). Energy Expenditure during

350 Functional Daily Life Performances in Patients with Fibromyalgia. Pain practice : the official journal of World Institute of Pain. doi: 10.1111/papr.12245

352

353

354

355

356

357

Lami, M. J., Martinez, M. P., Sanchez, A. I., Miro, E., Diener, F. N., Prados, G., \& Guzman, M. A. (2016). Gender Differences in Patients with Fibromyalgia Undergoing CognitiveBehavioral Therapy for Insomnia: Preliminary Data. Pain practice : the official journal of World Institute of Pain, 16(2), E23-34. doi: 10.1111/papr.12411

Lewis, G. N., \& Rosie, J. A. (2012). Virtual reality games for movement rehabilitation in neurological conditions: how do we meet the needs and expectations of the users? Disability and rehabilitation, 34(22), 1880-1886. doi: 10.3109/09638288.2012.670036

Mortensen, J., Kristensen, L. Q., Brooks, E. P., \& Brooks, A. L. (2015). Women with fibromyalgia's experience with three motion-controlled video game consoles and indicators of symptom severity and performance of activities of daily living. Disability and rehabilitation. Assistive technology, 10(1), 61-66. doi: $10.3109 / 17483107.2013 .836687$

Oliver, K., \& Cronan, T. (2002). Predictors of exercise behaviors among fibromyalgia patients. Preventive medicine, 35(4), 383-389.

Podsiadlo, D., \& Richardson, S. (1991). The timed" Up \& Go": a test of basic functional mobility for frail elderly persons. Journal of the American geriatrics Society, 39(2), 142148.

Rutledge, D. N., Martinez, A., Traska, T. K., \& Rose, D. J. (2013). Fall experiences of persons with fibromyalgia over 6 months. Journal of advanced nursing, 69(2), 435-448. doi: 10.1111/j.1365-2648.2012.06026.x 
372 Scheffer, A. C., Schuurmans, M. J., vanDijk, N., van der Hooft, T., \& de Rooij, S. E. (2010).

373 Reliability and validity of the visual analogue scale for fear of falling in older persons. Journal of the American Geriatrics Society, 58(11), 2228-2230. doi: 10.1111/j.15325415.2010.03105.x

376

Segura-Jimenez, V., Aparicio, V. A., Alvarez-Gallardo, I. C., Carbonell-Baeza, A., TorneroQuinones, I., \& Delgado-Fernandez, M. (2015). Does body composition differ between fibromyalgia patients and controls? the al-Andalus project. Clinical and experimental rheumatology, 33(1 Suppl 88), S25-32.

Skip Rizzo, A., Lange, B., Suma, E. A., \& Bolas, M. (2011). Virtual reality and interactive digital game technology: new tools to address obesity and diabetes. Journal of diabetes science and technology, 5(2), 256-264.

Tomas-Carus, P., Gusi, N., Hakkinen, A., Hakkinen, K., Raimundo, A., \& Ortega-Alonso, A. (2009). Improvements of muscle strength predicted benefits in HRQOL and postural balance in women with fibromyalgia: an 8-month randomized controlled trial. Rheumatology, 48(9), 1147-1151. doi: 10.1093/rheumatology/kep208

van Diest, M., Stegenga, J., Wortche, H. J., Verkerke, G. J., Postema, K., \& Lamoth, C. J. (2016). Exergames for unsupervised balance training at home: A pilot study in healthy older adults. Gait \& posture, 44, 161-167. doi: 10.1016/j.gaitpost.2015.11.019

Walitt, B., Nahin, R. L., Katz, R. S., Bergman, M. J., \& Wolfe, F. (2015). The Prevalence and Characteristics of Fibromyalgia in the 2012 National Health Interview Survey. PloS one, 10(9), e0138024. doi: 10.1371/journal.pone.0138024

Walker, J. (2016). Fibromyalgia: clinical features, diagnosis and management. Nursing standard, 31(5), 51-63. doi: 10.7748/ns.2016.e10550 
395 Wolfe, F., Clauw, D. J., Fitzcharles, M. A., Goldenberg, D. L., Katz, R. S., Mease, P., . . Yunus, 396 M. B. (2010). The American College of Rheumatology preliminary diagnostic criteria for fibromyalgia and measurement of symptom severity. Arthritis care \& research, 62(5), 600-610. doi: 10.1002/acr.20140

399 Wolfe, F., Smythe, H. A., Yunus, M. B., Bennett, R. M., Bombardier, C., Goldenberg, D. L., .. . 400 Sheon, R. P. (1990). The American College of Rheumatology 1990 Criteria for the 401 Classification of Fibromyalgia. Report of the Multicenter Criteria Committee. Arthritis

402 and rheumatism, 33(2), 160-172. 


\section{Table $\mathbf{1}$ (on next page)}

Baseline characteristics of the study participants

EG, Exercise group; CG, Control group. TUG: Timed-Up-Go. EOFS, eyes open on a firm surface; ECFS, eyes closed on a firm surface; EOUS, eyes open on an unstable surface; ECUS, eyes closed on an unstable surface. BMI, Body Mass Index. 
Table 1. Baseline characteristics of the study participants

\begin{tabular}{|c|c|c|}
\hline Variable (mean, SD) & EG $(n=41)$ & CG $(n=35)$ \\
\hline Age (years) & $52.43(9.83)$ & $52.58(9.42)$ \\
\hline \multicolumn{3}{|l|}{ Age distribution } \\
\hline$\leq 40($ frequency and $\%)$ & $4(9.8 \%)$ & $3(8.6 \%)$ \\
\hline 41 to 60 (frequency and \%) & $28(68.3 \%)$ & $24(68.6 \%)$ \\
\hline$\geq 60($ frequency and $\%)$ & $9(22 \%)$ & $8(22.9 \%)$ \\
\hline Years since diagnosis (years) & $10.36(7.30)$ & $12.48(5.63)$ \\
\hline BMI $\left(\mathrm{kg} / \mathrm{m}^{2}\right)$ & $25.79(5.14)$ & $27.75(5.62)$ \\
\hline Waist:hip ratio & $0.85(0.07)$ & $0.86(0.06)$ \\
\hline FIQ-R & $46.21(16.49)$ & $43.64(18.18)$ \\
\hline
\end{tabular}

EG, Exercise group; CG, Control group.

BMI, Body Mass Index; SD, Standard deviation.

FIQ-R, Revised version of the fibromyalgia impact questionnaire.

1

2

3

4 


\section{Table 2 (on next page)}

Effects of the 8-week training program on the VirtualEx-FM exergame and control groups $(n=76)$

EG, Exercise group; CG, Control group. TUG: Timed-Up-Go. EOFS, eyes open on a firm surface; ECFS, eyes closed on a firm surface; EOUS, eyes open on an unstable surface; ECUS, eyes closed on an unstable surface. ap-values calculated using paired samples t-test; ${ }^{\mathrm{b} P}$ values calculated using analysis of variance (ANOVA) for repeated measures (to compare differences between groups after the 8-week training program). 
Table 2. Effects of the 8-week training program on the VirtualEx-FM exergame and control groups $(n=76)$

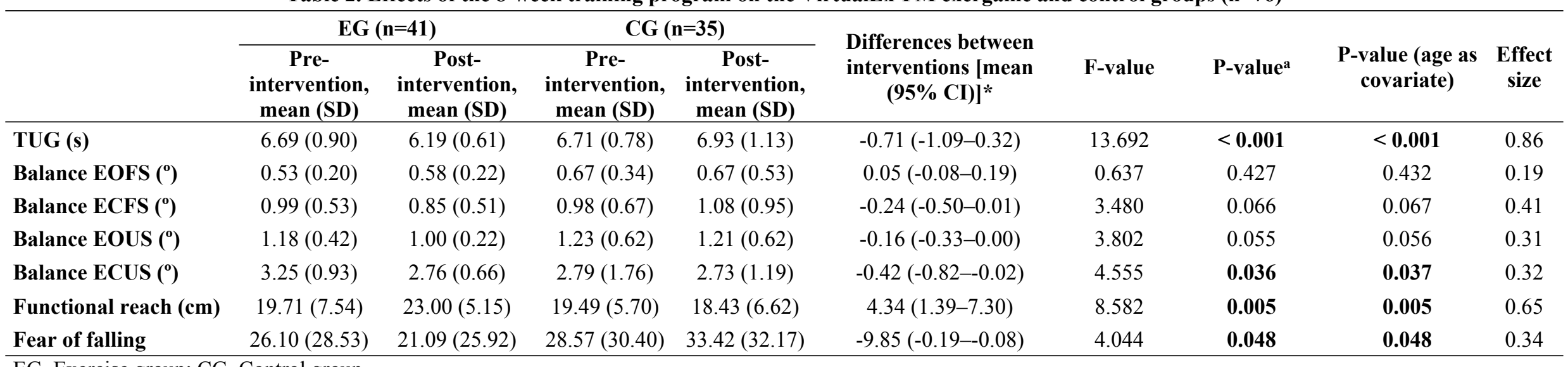

EG, Exercise group; CG, Control group.

TUG: Timed-Up-Go.

EOFS, eyes open on a firm surface; ECFS, eyes closed on a firm surface; EOUS, eyes open on an unstable surface; ECUS, eyes closed on an unstable surface.

*Differences between interventions: calculated as "(post-intervention EG" - "pre-intervention EG) - (post-intervention CG" - "pre-intervention CG)

aP-values calculated using analysis of variance (ANOVA) for repeated measures; SD, Standard deviation. 


\section{Table 3(on next page)}

Effects of VirtualEx-FM on fear of falling in the exergame and control groups $(n=76)$

EG, Exercise group; CG, Control group. aP-values calculated using the paired samples t-test; ${ }^{b} p$-values calculated using analysis of variance (ANOVA) for repeated measures (to compare differences between groups after the 8-week training program). 
Table 3. Effects of VirtualEx-FM on fear of falling in the exergame and control groups $(n=76)$

\begin{tabular}{|c|c|c|c|c|c|c|c|}
\hline & \multicolumn{2}{|c|}{ Fearless EG (fear of falling $<50 ; n=28$ ) } & \multicolumn{2}{|c|}{ Fearless CG (fear of falling $<50 ; n=27$ ) } & \multirow{2}{*}{$\begin{array}{l}\text { Difference between } \\
\text { intervention (virtual } \\
\text { minus control)* }\end{array}$} & \multirow{2}{*}{$\begin{array}{l}\text { P-value } \\
\text { interaction }^{\text {a }}\end{array}$} & \multirow{2}{*}{ Effect size } \\
\hline & $\begin{array}{l}\text { Pre-intervention, } \\
\text { mean (SD) }\end{array}$ & $\begin{array}{l}\text { Post-intervention, } \\
\text { mean (SD) }\end{array}$ & $\begin{array}{l}\text { Pre-intervention, } \\
\text { mean (SD) }\end{array}$ & $\begin{array}{l}\text { Post-intervention, } \\
\text { mean (SD) }\end{array}$ & & & \\
\hline \multirow[t]{3}{*}{ Fear of falling } & $8.75(12.29)$ & $10.53(18.97)$ & $14.44(14.76)$ & $21.85(25.87)$ & $-5.62(-16.09-4.85)$ & 0.287 & 0.28 \\
\hline & \multicolumn{2}{|c|}{ Fearful EG (fear of falling $>50 ; n=13$ ) } & \multicolumn{2}{|c|}{ Fearful CG (fear of falling $>50 ; n=8$ ) } & Differences between & P-value & Effect size \\
\hline & $\begin{array}{l}\text { Pre-intervention, } \\
\text { mean (SD) }\end{array}$ & $\begin{array}{l}\text { Post-intervention, } \\
\text { mean (SD) }\end{array}$ & $\begin{array}{l}\text { Pre-intervention, } \\
\text { mean (SD) }\end{array}$ & $\begin{array}{l}\text { Post-intervention, } \\
\text { mean (SD) }\end{array}$ & $\begin{array}{l}\text { interventions (virtual } \\
\text { minus control) }\end{array}$ & Interaction & \\
\hline Fear of falling & $63.46(12.64)$ & $43.84(24.67)$ & $76.25(17.67)$ & $72.50(16.69)$ & $-15.86(-36.17-4.44)$ & 0.119 & 1.13 \\
\hline
\end{tabular}

EG, Exercise group; CG, Control group.

SD, Standard deviation.

*Differences between interventions: calculated as "(post-intervention EG" - "pre-intervention EG) - (post-intervention CG" - "pre-intervention CG)

${ }^{a} \mathrm{P}$-values calculated using analysis of variance (ANOVA) for repeated measures; SD, Standard deviation; SD, Standard deviation. 


\section{Figure 1 (on next page)}

Flow of participants 


\section{CONSORT 2010 Flow Diagram}

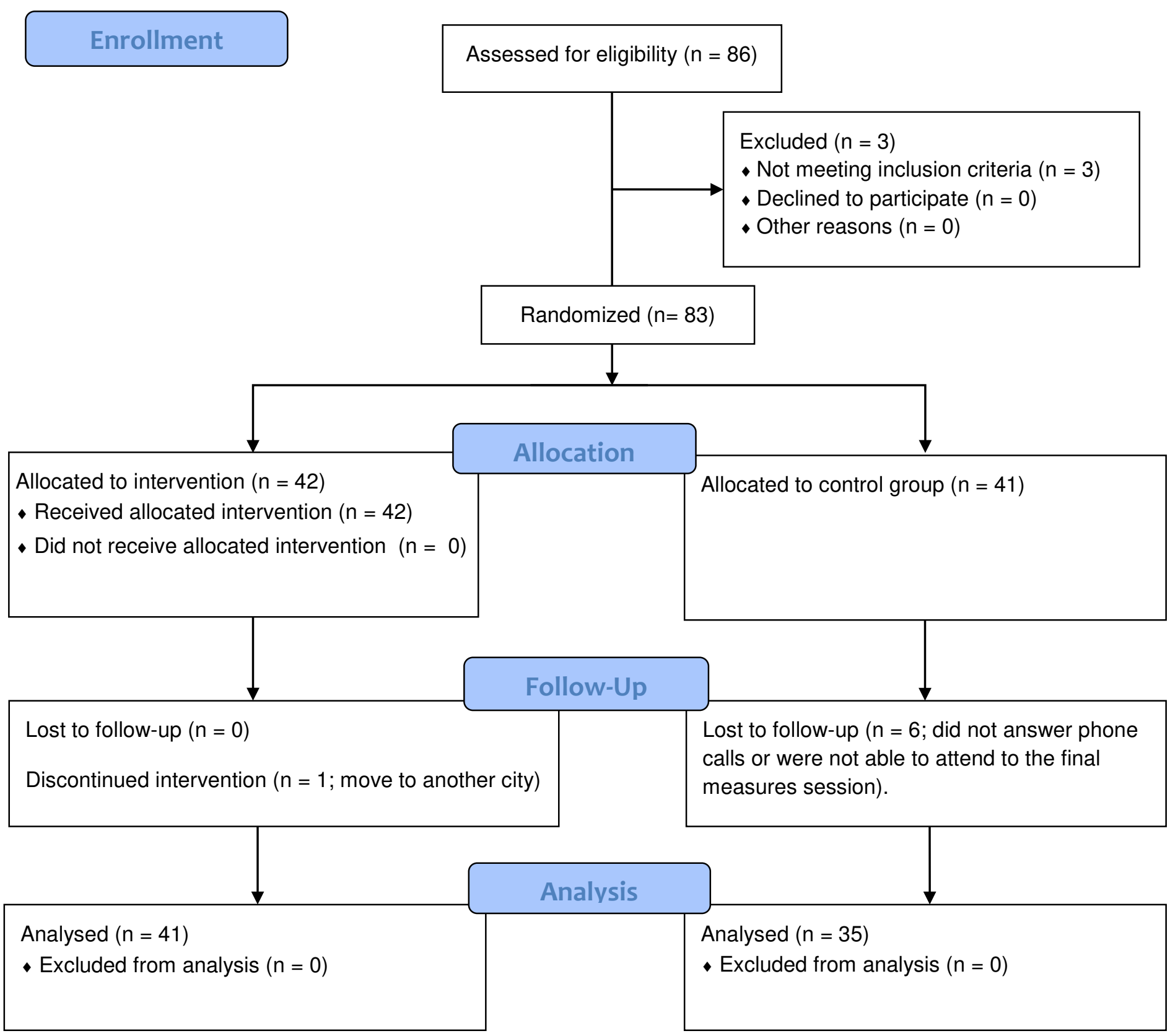

